\section{APRENDIZAJE Y CONOCIMIENTO PARA INNOVAR Y EMPRENDER}

\author{
José Luis López de Silanes \\ Presidente Ejecutivo del Grupo CLH \\ Presidente del Consejo Social de la Universidad de La Rioja
}

\section{LEARNING AND KNOWLEDGE FOR INNOVATION AND ENTREPRENEURSHIP}

ABSTRACT: Learning and the creation of knowledge are closely related to improved competitiveness and the creation of wealth in a country, because they help to boost innovation and the development of more productive activities with higher added value by taking advantages of the opportunities offered by the new technologies.

Spain, which has made a significant effort during the last 30 years and has managed not only to position itself in ninth place among the countries with the highest scientific production, but also among the major world economies, has lost momentum in recent years, which is why it needs to improve its competitive capacity.

In order to achieve this, an appropriate environment must be generated through promoting the innovative capacity of all the institutions involved and improving the quality of education, as well as the conditions required for facilitating the free movement of knowledge.

In this context, the Spanish university still has an important task before it in terms of improving optimization of the use made of its educational resources and scientific production, as well as the continuous updating of knowledge and promotion of the entrepreneurial ability of young university students, with the assistance of society as a whole.

KEY WORDS: Learning; knowledge; innovation; entrepreneurship; competitiveness; university.

\section{INTRODUCCIÓN}

Durante un reciente encuentro que mantuvimos en $\mathrm{CLH}$ con el Presidente de IBM en España, Juan Antonio Zufiria, dedicado a hablar de la innovación y la excelencia, el Presidente de IBM puso como ejemplo de los recientes logros conseguidos por su empresa, la creación de un superordenador, denominado Watson.

Una de las principales innovaciones de este ordenador consiste en que es capaz de desenvolverse con gran naturalidad en entornos de preguntas y respuestas, entendiendo el contenido de las mismas y ofreciendo respuestas acertadas en muy poco tiempo.
RESUMEN: El aprendizaje y la creación de conocimiento están estrechamente relacionados con la mejora de la competitividad y la creación de riqueza de un país, porque contribuyen a potenciar la innovación y el desarrollo de actividades más productivas y de mayor valor añadido aprovechando las oportunidades que brindan las nuevas tecnologías.

España, que ha realizado un importante esfuerzo durante los últimos 30 años, y ha logrado situarse entre las principales economías del mundo, ha perdido posiciones en los últimos años, por lo que necesita mejorar su capacidad competitiva.

Para lograrlo, es necesario generar un entorno adecuado mediante el fomento de la capacidad innovadora de todas las instituciones implicadas y la mejora de la calidad educativa, asi como de las condiciones necesarias para facilitar la libre circulación del conocimiento.

Dentro de este contexto, la universidad española todavía tiene una importante tarea de mejora en la optimización de la utilización de sus recursos educativos y de su producción científica, así como en la actualización continua del conocimiento y en fomentar la capacidad emprendedora de los jóvenes universitarios, con la colaboración del conjunto de la sociedad.

PALABRAS CLAVE: Aprendizaje; conocimiento; innovación; emprender; competitividad; universidad.

Esta capacidad le permite competir con seres humanos en concursos televisivos de preguntas y respuestas.

En su intervención el Presidente de IBM destacó la dificultad que supone para cualquier ordenador entender el lenguaje natural humano, debido a la cantidad de información sobreentendida que expresamos cada vez que hablamos.

En este sentido destacó la gran innovación que supone tener ordenadores capaces de realizar una tarea tan difícil como es entender lo que decimos, lo que permitirá aplicarlo a muy distintos usos, incluido el diagnóstico de enfermedades. 
Por esta razón, y con el objetivo de enfocar lo más certeramente posible el contenido de este artículo, acudí al Diccionario de la Real Academia, para precisar más claramente los distintos conceptos a los que hace referencia: aprendizaje, innovación, competitividad, y conocimiento.

Después de leer las definiciones del Diccionario de la Real Academia, no estoy seguro de que ni siquiera un superordenador como el que ha diseñado IBM, fuera capaz de entender y relacionar con claridad dichos conceptos, ya que a diferencia de los seres humanos, los ordenadores, incluso los más potentes, no son todavía capaces de aprender ni generar auténtico conocimiento.

En cambio estoy seguro de que todos conocemos y compartimos la gran importancia y trascendencia que tienen para nosotros el aprendizaje, la innovación, la competitividad y el conocimiento, siempre y cuando no tengamos que explicarlo.

\section{Definiciones}

Según la definición del Diccionario de la Real Academia, el "aprendizaje" es "la acción y efecto de aprender algún arte, oficio u otra cosa". Esta definición un tanto circular nos obliga a buscar el significado del verbo aprender, que según la RAE es "adquirir el conocimiento de algo por medio del estudio o de la experiencia".

Asimismo, el Diccionario de la RAE define la "innovación" como "acción y efecto de innovar", entendiendo por innovar "mudar o alterar algo introduciendo novedades".

En cuanto al término "competitividad", el Diccionario de la RAE dice que es "la capacidad de competir".

A su vez, la palabra "competir" tiene dos acepciones posibles: "dicho de dos o más personas: contender entre sí aspirando a una misma cosa" y "dicho de una cosa: igualar a otra análoga en la perfección o en las propiedades".

Por último, si buscamos el término "conocimiento", la definición que nos da el Diccionario de la Real Academia es "acción y efecto de conocer", lo que nos obliga de nuevo a buscar qué entiende la Real Academia por "conocer".

Lo que encontramos en este caso tiene bastante que ver con la definición que daba de aprender: "averiguar por el ejercicio de las facultades intelectuales la naturaleza, cualidades y relaciones de las cosas". Si bien en otra de las acepciones de conocimiento la RAE también menciona "noción, ciencia, sabiduria".

\section{LIBERTAD DE CIRCULACIÓN DEL CONOCIMIENTO}

Para el gran filósofo inglés del siglo XVI, Francis Bacon, "el conocimiento es poder".

Trasladándolo a nuestro mundo actual y en el ámbito del contenido de este artículo, esto significa que a través del aprendizaje podemos lograr el poder que nos proporciona el conocimiento, y utilizarlo para seguir innovando y compitiendo, en un mundo cada vez más globalizado y más complejo.

Siguiendo esta línea de pensamiento, me gustaría introducir una breve reflexión para el debate, en relación con el concepto de "la libre circulación del conocimiento".

Porque, si identificamos el conocimiento con el poder, como hacía el filósofo Francis Bacon ¿cómo vamos a estar interesados en que circule libremente?

¿Por qué compartir ese poder que tan arduamente hemos logrado aplicando nuestra capacidad de aprendizaje?

¿Por qué vamos a compartir el conocimiento, si lo que queremos es mejorar nuestra capacidad competitiva a través de la innovación?

El motivo fundamental que se me ocurre para que estemos interesados en fomentar la circulación del conocimiento, es que a diferencia de cualquier otro bien que pueda crear el ser humano, compartir el conocimiento no significa vernos privados de él.

Más bien al contrario, ya que compartir el conocimiento nos da la oportunidad de que otras personas incrementen 
el saber que nosotros hemos contribuido a crear, porque como decía Séneca, los hombres aprenden mientras enseñan.

De este modo, si establecemos sistemas adecuados para compartir el conocimiento, protegiendo debidamente la propiedad intelectual y los derechos relacionados con la misma, se puede llegar a generar una espiral de creación de conocimiento.

Y así contribuiremos a construir un mundo cada vez más inteligente que nos ayude a resolver los grandes retos a los que se enfrenta la humanidad en la actualidad.

\section{LIBERTAD DE CONOCIMIENTO Y PROPIEDAD INTELECTUAL}

En este contexto, la situación ideal sería que una vez realizado un descubrimiento, los nuevos conocimientos estuvieran a disposición de todo el mundo, y que la sociedad pudiera hacer el mejor uso posible de los mismos.

Sin embargo, es indudable que la generación de conocimiento ha adquirido una complejidad tal que cada vez resulta más difícil diferenciar entre conocimiento básico y aplicaciones.

Por esta razón, en muchas ocasiones no se puede determinar claramente qué conocimientos pertenecen a la categoría de bien público sujeto a la libertad académica y la libre circulación, y qué conocimientos deben ser protegidos con patentes o incluso sometidos a restricciones de secreto industrial o de estado.

Un ejemplo de ello es la cada vez más estrecha relación que existe actualmente entre los descubrimientos en biología o física y sus aplicaciones en la industria química y mecánica respectivamente.

También hay que tener en cuenta que muchas disciplinas requieren grandes inversiones para crear conocimiento, por lo que es lógico que los organismos o las entidades que proporcionan esos recursos, traten de garantizarse la recuperación de la inversión, protegiendo de algún modo el conocimiento que han contribuido a crear.
Este dilema es especialmente evidente en el caso de las universidades y centros de investigación de carácter público, lo que ha supuesto que en distintos países se tomen medidas para tratar de facilitar la circulación del conocimiento, garantizando al mismo tiempo que las entidades que realizan los descubrimientos puedan ser titulares de patentes o derechos de propiedad intelectual que les permitan seguir financiando su labor científica.

Un ejemplo de ello es la Ley Bayh-Dole del Congreso de los Estados Unidos, promulgada en 1980, que permite a las universidades norteamericanas patentar y explotar comercialmente sus descubrimientos, si bien está siendo objeto de cierta polémica porque desde algunos ámbitos se considera que crea obstáculos al progreso de la investigación.

Del mismo modo, con motivo del relanzamiento de la Estrategia de Lisboa, la Comisión Europea publicó en abril de 2008 una recomendación sobre la gestión de la propiedad intelectual en las actividades de transferencia de conocimientos y un código de buenas prácticas para las universidades y otros organismos públicos de investigación.

La finalidad de esta Recomendación es alentar a los Estados miembros a que definan o adopten políticas $u$ orientaciones sobre la gestión de la propiedad intelectual y la transferencia de conocimiento.

Lo que resulta evidente, es que en este nuevo entorno que define la nueva sociedad de la información y la circulación global de las ideas nos enfrentamos a nuevos retos relacionados con la circulación del conocimiento que no se pueden resolver aplicando soluciones del pasado.

Esto implica la necesidad de abordar una reflexión sobre la libre circulación del conocimiento y la protección de la propiedad intelectual desde un enfoque multidisciplinar, y mucho más amplio que el que permiten los límites de este artículo.

\section{Creación de conocimiento y CREación DE RIQUEZA}

En un mundo como el de hoy, donde existe una gran interactividad y tenemos práctica inmediatez en las co-

ARBOR Vol. 187 Extra 3 diciembre [2011] 3-13 ISSN: 0210-1963 
municaciones podría parecer que la capacidad de creación de conocimiento está mucho más distribuida que en el pasado.

Sin embargo, si observamos algunos datos, comprobamos que los países que realizan el mayor esfuerzo en $I+D$ en comparación con su Producto Interior Bruto (PIB), siguen siendo países como Japón, Corea, Australia, Estados Unidos, Alemania, Francia, Canadá, Reino Unido, o España, que a su vez se sitúan también entre los países más ricos del mundo (Informe Cotec, 2011, p. 25).

Esta correlación parece poner de manifiesto que la creación de riqueza de un país se apoya fundamentalmente en su esfuerzo de inversión en investigación y desarrollo tecnológico, especialmente cuando sus economías ya han superado un determinado nivel.

Según la definición clásica de las etapas de desarrollo económico realizada por Michael Porter, en una primera fase las economías están soportadas por dos factores principales que son la mano de obra no cualificada y los recursos naturales.

Esto explicaría por qué un país emergente como China, cuya economía está creciendo de forma espectacular, y podría desbancar a Estados Unidos de la primera posición mundial, no aparece todavía en las primeras posiciones de los ranking de inversión en $\mathrm{I}+\mathrm{D}$, en proporción a su Producto Interior Bruto.

Aplicando la teoría de Porter, China todavía estaría apurando esa etapa en la que las economías están soportadas por la utilización de mano de obra barata y recursos naturales, con una capacidad competitiva basada fundamentalmente en los precios.

En este sentido, no parece posible que la economía china continúe creciendo indefinidamente a una tasa anual del $8 \%$ o el 10\%, como ha venido ocurriendo durante los últimos años, si no es capaz de pasar a una nueva etapa más evolucionada.

En esa nueva etapa, siguiendo a Porter, el impulso para el crecimiento proviene principalmente de la mejora de la eficiencia y la calidad, lo que daría paso a la tercera etapa, que es la de la innovación.
Y de hecho parece que China lo está haciendo así. A pesar de que el porcentaje de inversión en $\mathrm{I}+\mathrm{D}$ en relación con su Producto Interior Bruto es todavía relativamente bajo en términos comparativos, su producción científica ha aumentado más del 260\% entre 1999 y 2008.

Este importante nivel de crecimiento coloca a China en el segundo puesto como productor de conocimiento científico en volumen absoluto.

Siguiendo de nuevo la teoría de Porter, en la etapa de la innovación, que es en la que se encontrarían la mayoría de los países desarrollados que citábamos antes, y que próximamente también podría alcanzar China, sólo es posible mantener el nivel de crecimiento económico si se es capaz de competir con productos y servicios únicos y cada vez más innovadores.

\section{Factores de competitividad}

Según el Foro Económico Mundial, que analiza desde 1979 los factores que permiten a las economías nacionales alcanzar un crecimiento económico, la competitividad se basa en 12 pilares, que se organizan a su vez en tres bloques.

El primero de ellos está constituido por los requerimientos básicos de cualquier economía, y agrupa los pilares siguientes: instituciones, infraestructura, estabilidad macroeconómica, salud y educación primaria.

El segundo de los bloques está formado por los factores potenciadores de la eficiencia. Esto incluye la eficiencia en el mercado de bienes, la eficiencia en el mercado laboral, la sofisticación del mercado financiero, la disponibilidad tecnológica, el tamaño del mercado y la educación superior y el aprendizaje.

Finalmente, el tercer bloque se refiere a los factores de innovación y sofisticación, que incluyen la sofisticación de negocio y la innovación.

Como se puede ver claramente, los doce pilares son interdependientes y tienden a reforzarse. Así, por ejemplo, la innovación es difícil si el nivel de educación es bajo y la fuerza laboral no tiene la formación adecuada.

Además, la innovación es improbable en un entorno sin instituciones que garanticen los derechos de propiedad 
intelectual, o los mercados son ineficientes y no hay infraestructuras extensas y adecuadas.

\section{Posición competitiva e innovación en España}

Desde que el Foro Económico Mundial aplicó por primera vez el concepto de los doce pilares de la competitividad a España, la posición de nuestro país se ha deteriorado en los tres aspectos considerados.

Este deterioro ha sido especialmente importante en los requerimientos básicos, aspecto en el que bajó del puesto 25 al 38, y en factores de innovación y sofisticación, en el que pasó del puesto 30 al 41 (Informe Cotec, 2911, pp. 90-91).

Aunque según el último ranking publicado en 2011, España ha logrado recuperar parte de la competitividad perdida por la crisis económica y ha logrado ascender hasta el puesto 36 , de una lista de 142, esta posición sigue siendo baja para un país que antes del comienzo de la actual crisis económica era la novena economía del mundo.

$Y$ es especialmente bajo, si consideramos además, que España aspira realizar un cambio de su modelo económico hacia otro modelo basado en la innovación, y orientado hacia actividades más productivas, de mayor valor añadido y que aproveche las oportunidades que brindan las nuevas tecnologías.

Sin embargo, es preciso reconocer el gran esfuerzo que ha realizado nuestro país durante los últimos 20 ó 30 años, destinando cada vez más recursos a la investigación, lo que ha permitido situar a España en el noveno puesto de los países con mayor producción científica del mundo (Informe CYD, 2010, p. 159).

Actualmente, el desarrollo de este esfuerzo se concentra principalmente en tres ámbitos, que son: las instituciones públicas de investigación, como el CSID y el CDTI, así como las universidades, y las empresas.

Según las estadisticas sobre las actividades de investigación científica y desarrollo tecnológico que elabora el Instituto Nacional de Estadística, la inversión en I+D rea- lizada en España en 2009 fue de casi quince mil millones de euros.

Más del 50\% correspondieron a las inversiones realizadas por las empresas y las instituciones privadas, cerca del $28 \%$ a las universidades, y el 20\% a la Administración Pública (Informe Cotec, 2011, pp. 21-22).

A pesar de este notable esfuerzo, la posición de España, en cuanto a competitividad e innovación sigue siendo de retraso respecto a tres grandes países de la Unión Europea (Alemania, Francia y Reino Unido), y también respecto a Japón, Estados Unidos y otros países industrializados.

Además, hay que tener en cuenta que la propia Unión Europea está retrocediendo posiciones en materia de competitividad dentro del panorama mundial.

El ex primer ministro de Reino Unido, Gordon Brown, alertaba hace unos meses que Europa posee una tasa de crecimiento tendencial que es la mitad de la de Estados Unidos y un cuarto de la de China e India.

Observando estos datos, Gordon Brown señalaba el riesgo de que si el crecimiento europeo sigue así de retrasado respecto al de sus competidores, a mitad de siglo la economía europea podría tener la misma dimensión que la de África.

\section{Fomento de LA INNOVACIÓN}

En un tono algo menos dramático que el del ex primer ministro británico, la Comisión Europea ya había señalado en octubre de 2010 algunas limitaciones del sistema de innovación europeo, que podrian estar lastrando el crecimiento del viejo continente como son:

- Inversión insuficiente en nuestra base de conocimiento, frente a otros países, como Estados Unidos y Japón, que invierten más que Europa, o incluso China que se está acercando rápidamente.

- Condiciones marco insatisfactorias, que van desde el acceso deficiente a la financiación, pasando por los costes elevados de los derechos de propiedad intelectual e industrial, hasta la lenta normalización y el uso ineficaz de la contratación pública. 
- Fragmentación excesiva y duplicaciones costosas, que impiden aplicar recursos de una manera más eficiente y alcanzar una masa crítica.

Para lograr superar esta situación, la Comisión Europea presentó el proyecto denominado: "Unión por la innovación", con el que trata de convertir la innovación en un objetivo de actuación general a medio y largo plazo.

Según este planteamiento se pretende que los instrumentos, las medidas y la financiación de las políticas estén diseñados de modo que contribuyan a la innovación.

Asimismo se busca que las políticas de la Unión Europea y las políticas nacionales y regionales estén en consonancia y se refuercen mutuamente, y que se establezca una agenda estratégica, que facilite el seguimiento regular de los avances.

\section{Futuro de la inNovación en España}

También España, como miembro activo de la Unión Europea, está intentando sentar las bases de un desarrollo más sostenible, mediante el fomento de la capacidad innovadora y la mejora de la calidad de la educación.

En este marco se inscriben la reciente aprobación de la Ley de Economía Sostenible y la Ley de la Ciencia, la Tecnología y la Innovación.

La Ley de Economía Sostenible, aprobada en marzo de 2011, recoge expresamente la mejora de la competitividad, el fomento de la capacidad innovadora de las empresas y la extensión y mejora de la calidad de la educación, entre los principios que guiarán la acción de los poderes públicos para impulsar la sostenibilidad de la economía española.

Entre los capítulos más relevantes de esta Ley para lograr este objetivo, está el capítulo IV, dedicado al impulso de la sociedad de la información, y aún más el capítulo $V$, centrado en el fomento de la actividad investigadora y la innovación.

La primera sección de este capítulo se dedica a la transferencia de los resultados de la actividad investigadora, fa- cilitando que los centros públicos de investigación puedan transferir conocimientos al sector privado.

Asimismo, la Ley insiste en el fomento de la cooperación entre los agentes públicos y privados a través de la participación en empresas innovadoras de base tecnológica.

Para ello, se facilita el que los organismos públicos de investigación, las universidades y otros agentes relevantes puedan participar en el capital de empresas relacionadas con la investigación y con el aprovechamiento industrial o comercial de las innovaciones.

También la segunda sección de este capítulo de la Ley abunda en aspectos relacionados con aspectos vinculados a la innovación.

En concreto, hay varios artículos dedicados a la formación, investigación y transferencia de resultados, que deberán atender a la consecución de una serie de objetivos ligados a un esfuerzo de modernización, mejora de la calidad y búsqueda de la excelencia académica.

Por su parte, la Ley de la Ciencia, la Tecnología y la Innovación, que tiene el mérito adicional de haber sido aprobada con un amplio consenso entre los grupos parlamentarios, reconoce que la apuesta por la innovación es estrictamente necesaria para el crecimiento y competitividad de nuestro sistema productivo.

Igualmente señala que la economía española debe avanzar hacia un modelo productivo en el que la innovación está llamada a incorporarse definitivamente como una actividad sistemática de todas las empresas con independencia de su sector y tamaño.

Así, la Ley define como objetivo fundamental la promoción de la investigación, el desarrollo experimental y la innovación como elementos sobre los que ha de asentarse el desarrollo económico sostenible y el bienestar social.

Sin embargo, la Ley también es muy realista al reconocer la diferencia sustancial que existe entre la intervención pública que requiere el fomento de la investigación, incluida la investigación científica y técnica que realizan las empresas, y la creación de un entorno favorable a la innovación, que constituye un reto mucho más transversal. 


\section{ApREndizAJE Y COMPETITIVIDAD}

En esta línea, no sólo necesitamos contar con un adecuado nivel de producción científica, sino también con un tejido empresarial que sea capaz de aprovechar las fuentes de conocimiento y tecnología a su alcance para producir productos y servicios innovadores.

Igualmente será necesario contar con un capital humano cada vez más capacitado para adquirir los conocimientos precisos y desarrollar las tecnologías adecuadas.

En este contexto, comprobamos de nuevo la gran importancia del aprendizaje y la circulación del conocimiento para la mejora de la competitividad.

Una tarea en la que hay que destacar el importante papel que pueden desempeñar las empresas, y por supuesto las universidades, mediante la formación del capital humano, que constituye un elemento básico en este nuevo entorno de la sociedad del conocimiento.

Los análisis basados en datos históricos demuestran que la mejora en las habilidades cognitivas de una población a través de la educación acaba teniendo un impacto positivo en el crecimiento económico.

Esto no quiere decir que la calidad del sistema educativo sea el único factor de relevancia, ya que como hemos visto también es preciso que se den otra serie de factores para que un pais sea capaz de seguir progresando.

Sin embargo, es indudable que invertir en educación, si se invierte bien, es un buen negocio en términos económicos.

Pero tenemos que recordar, que como decía Unamuno, aunque el saber no ocupa lugar "el aprender ocupa tiempo, y mientras se aprende una cosa podría aprenderse otra de más sustancia".

\section{El papel de la Universidad}

En esta línea la Universidad española todavía tiene una importante tarea de mejora, como ponen de manifiesto los datos recogidos en el último informe de la Fundación CYD.
Según este informe, el sistema universitario español destina todavía una considerable cantidad de recursos al soporte de titulaciones con baja o muy baja demanda.

Esto implica una asignación muy poco productiva de los recursos totales asignados a la función docente de las universidades.

En concreto, según el último informe de CYD más del 42\% de las enseñanzas de nuestro sistema universitario tiene menos de 50 alumnos de nuevo ingreso (Informe CYD, 2010, p. 124).

Una situación similar se da en los títulos de máster, con una demanda académica por enseñanza de 26 alumnos (Informe CYD, 2010, p. 124).

El informe subraya también que nuestro sistema universitario está lejos de una situación de óptimo rendimiento en la utilización de los recursos para la producción científica.

Más del 24\% de la plantilla del personal docente e investigador con relación laboral de funcionarios no produce resultados científicos que hayan podido ser evaluados y reconocidos (Informe CYD, 2010, p. 129).

Además, el índice de relevancia de la producción científica que se realiza está en general a una distancia de más del $20 \%$ de los valores del entorno europeo, y de más del $50 \%$ respecto a los valores del Reino Unido y Estados Unidos (Informe CYD, 2010, p. 129).

Podríamos seguir concentrándonos en los problemas, muchos de ellos conocidos, pero creo que es preferible que nos concentremos en lo mucho que ha logrado nuestro país en estos últimos 50 años.

Durante este período, nuestro país ha conseguido triplicar su peso en el total de la economía europea, y ha conseguido salir reforzado de cada una de las crisis por las que ha pasado en las décadas de los 70, 80 y 90 (Informe Consejo Empresarial para la Competitividad, 2011, p. 8).

Un logro, en el que hay que reseñar la importante contribución que ha tenido la universidad en este gran desarrollo, así como lo mucho que todavía puede seguir haciendo. 
Resulta especialmente destacable el esfuerzo inversor realizado en educación durante todos estos años, lo que nos ha situado con un 39\% de la población entre 25 y 34 años con educación terciaria, lo que supone cuatro puntos porcentuales por encima de la media de los países de la Unión Europea de los 15, destacando la formación científica de primer nivel mundial y el prestigio de las escuelas de negocio de España (Informe Consejo Empresarial para la Competitividad, 2011, p. 22).

En esta línea, un aspecto importante en el que tenemos que seguir trabajando es el de promover y fomentar la actitud emprendedora de los estudiantes universitarios.

\section{AprendizAJe Y eMPLEO}

Durante una reciente visita a España, el Presidente del Consejo Nacional de Innovación de la India, y uno de los artífices de su gran despegue económico, Sam Pitroda, señaló que en el siglo XXI los jóvenes no deben salir de la universidad buscando un empleo.

Según él, eso es algo que pertenece al siglo pasado y no al actual, y afirmó que los jóvenes deberían salir de la universidad empeñados en crear empleo.

Si nos atenemos a los datos de España, la situación actual es justo la contraria, porque la mayoría de las pequeñas y medianas empresas que se crean en nuestro país las constituyen personas que no han pasado por la universidad o que la han abandonado antes de obtener un título universitario.

Otro de los aspectos que señalaba el responsable de innovación de la India, es que en todas partes faltan expertos en algo.

El problema en Estados Unidos y en Europa es que hay mucha gente formada que no encaja con la demanda real de empleos, porque hay un error en la planificación educativa.

Aunque es posible que desde su lejana posición en la India, este experto en innovación no conozca con todo detalle la situación de España, lo cierto es que su análisis se aplica perfectamente a nuestro país.
A pesar de que España soporta una de las tasas de paro más altas de toda Europa, con más de un 21\%, todavía hay puestos de trabajo para los que es difícil encontrar personas con la formación y la experiencia necesaria.

Al mismo tiempo, en los últimos meses, se han publicado algunos informes en los que se afirma que España cuenta con una juventud sobreeducada que no se adapta a las necesidades de nuestro mercado laboral.

Según datos de la Organización para la Cooperación y el Desarrollo Económico (OCDE) en torno al 40\% de los jóvenes españoles entre 25 y 29 años con titulación superior ocupan un puesto de trabajo de un rango profesional por debajo del correspondiente a su nivel educativo, frente al 23\% de media de la OCDE (Panorama de la Educación Indicadores de la OCDE, 2010, p. 20).

Además, recientemente sonaron las alarmas ante la noticia de que muchos ingenieros españoles tenían que emigrar a Alemania en busca de trabajo.

Y es que la dificultad para encontrar empleo ha hecho que en los últimos tres años más de 200.000 personas mayoritariamente jóvenes hayan abandonado el país.

Desde mi punto de vista, lo más importante en una situación de crisis como la actual, es que España necesita nuevos emprendedores capaces de ayudar a cambiar nuestro modelo de crecimiento hacia un modelo de mayor valor añadido.

Tratar de responsabilizar de esta tarea exclusivamente a la universidad constituye una simplificación excesiva y no ayudaria a resolver el problema.

Si queremos desarrollar una cultura más emprendedora e innovadora, la universidad tendrá que desempeñar un papel muy importante adecuando la formación académica a las nuevas necesidades que conlleva pasar de la "sociedad del ladrillo" a la "sociedad del conocimiento".

Pero es indudable que también los estudiantes tendrán que cambiar la manera de enfocar su paso por la universidad y decidir si eso es lo que más les conviene.

Contra lo que pueda parecer a la vista de los informes ya citados, que hablan de sobreeducación de los jóvenes 
españoles, la solución a la crisis no puede ser nunca menos formación, sino más y mejor adaptada a las necesidades reales del país.

De hecho, las cifras muestran que las personas con estudios superiores siguen teniendo menores tasas de paro y un nivel de sueldo más elevado, incluso aunque ocupen puestos por debajo de su cualificación profesional.

Asimismo, es evidente que las personas mejor formadas pueden contribuir también de forma más eficaz a mejorar la competitividad de sus empresas.

Esto significa que tenemos que mejorar la planificación de las universidades para tratar de adecuar la formación académica al mundo laboral, y también hacer un esfuerzo para atraer a los mejores talentos a la Universidad.

Al mismo tiempo hay que seguir potenciando otras opciones educativas igualmente válidas, como la formación profesional, que no ha tenido hasta ahora en nuestro país la atención y el reconocimiento que se merece, para que también resulte atractiva para los futuros estudiantes.

Según datos de la OCDE, en España sólo un 35\% de alumnos elige la opción de la Formación Profesional, frente a un $45 \%$ de la media de la Unión Europea.

Además, miles de alumnos se quedan cada año sin plaza para hacer un ciclo medio de Formación Profesional, y muchos otros tienen que optar por cambiar los estudios elegidos o desplazarse a otros centros más alejados de sus domicilios.

En esta línea, el Ministerio de Educación se ha marcado un ambicioso objetivo para mejorar la Formación Profesional en España y lograr atraer a 200.000 nuevos estudiantes.

Precisamente esta parece ser una de las fórmulas de éxito de algunos países, como Alemania.

En este país, la formación profesional está basada en un sistema de aprendices que se forman en el trabajo beneficiándose del conocimiento de los trabajadores veteranos, lo que permite contar con un sistema de formación más orientado al empleo.
Siguiendo este modelo de éxito, el Gobierno español ha aprobado recientemente un nuevo contrato para la formación y el aprendizaje que permitirá a los jóvenes alternar sus estudios en un centro formativo con un trabajo remunerado en una empresa, que se espera que contribuya a mejorar la cualificación profesional de los jóvenes y su incorporación al mercado laboral.

\section{Aprender a emprender}

Pero por sí solo, esto no ayudará a cambiar la situación en España, si no desaparece también el miedo que parecen tener los universitarios españoles a emprender.

Según una reciente encuesta realizada por la Universidad Complutense de Madrid, el 41\% de los estudiantes desearía trabajar en una empresa privada cuando finalice sus estudios, y el 30\% aspira a obtener una plaza de funcionario de la administración pública. Sólo el $8 \%$ de los universitarios encuestados manifiesta interés en montar su propio negocio en el futuro. Estos datos contrastan vivamente con otros estudios publicados en Estados Unidos donde el $70 \%$ de los estudiantes americanos aspira a poder montar o emprender un negocio.

Por tanto, la clave está en saber qué podemos hacer para cambiar las creencias de una sociedad que, como recordaba recientemente el ex presidente del Gobierno, Felipe González, "no premia el mérito o la iniciativa con riesgo y castiga con crueldad el fracaso".

\section{Del modelo de enseñanza al modelo DE APRENDIZAJE}

Para terminar, y a modo de conclusión quisiera tratar de reformular algunas de las ideas que vimos al principio sobre aprendizaje, innovación, competitividad y conocimiento incorporando también otra que, como ya he comentado, resulta absolutamente imprescindible, que es la capacidad de emprender.

En un mundo en el que hay un exceso de información disponible, que crece exponencialmente, hay que replantearse 
en qué consiste el verdadero aprendizaje y la verdadera formación.

Tenemos que ser capaces de enseñar a los jóvenes de hoy a innovar no sólo en productos y servicios o en la organización de los procesos, sino incluso en la forma de enfocar su propia carrera profesional.

En este sentido también tenemos que enseñarles a seguir aprendiendo a lo largo de toda su carrera profesional para que sean capaces de adaptarse a un entorno crecientemente cambiante y competitivo.

Esto implica que la Universidad sea capaz de gestionar adecuadamente el cambio que supone la adopción de los acuerdos de Bolonia, para pasar de un modelo de enseñanza a uno de aprendizaje.

Este nuevo modelo significa que el alumno debe tomar una parte mucho más activa en el proceso educativo, por lo que los contenidos académicos deben circular de manera más fluida a través de las tecnologías de la información actualmente disponibles.

Asimismo, supone que el proceso de aprendizaje no puede considerarse acabado nunca, por lo que la universidad también puede, y debe, jugar un papel relevante en la actualización continua del conocimiento, y más aún en el entorno de rápida evolución que estamos viviendo.

\section{LA SOCIEDAD DEL CAMBIO}

De las 25 principales corporaciones industriales que existían en Estados Unidos en el año 1900, sólo dos continuaban en ese ranking en 1961. Y de las 25 primeras empresas que lideraban en 1962 el ranking Fortune 500, sólo cuatro seguían en él en 2010.

El ordenador personal se creó hace poco más de 40 años. La red que dio origen a internet hace algo más de 30 . El teléfono móvil que constituye una herramienta imprescindible para cualquiera de nosotros hace menos de 30 . $Y$ el buscador Google, sin el que ya no sabríamos trabajar hace menos de 20.
Durante estas últimas décadas los cambios han sido vertiginosos, y la tecnología ha avanzado deprisa, pero si creemos a los expertos, cada vez avanzará más rápidamente.

Se estima que más o menos cada tres o cinco años se duplica el conocimiento, casi el ritmo al que el Dr. Gordon Moore predijo que se duplicaría el número de transistores y la capacidad de memoria de un microprocesador, que se ha venido cumpliendo durante los últimos años.

Aunque la llamada "Ley de Moore" no es extrapolable a todas las áreas de conocimiento, es un hecho que se está produciendo una revolución en los límites del conocimiento en todas las ramas del saber, y una creciente tecnificación de nuestra sociedad.

Para poner sólo un ejemplo, cuando hoy nos ponemos al volante de un coche de gama media, estamos al mando de una máquina tecnológicamente más compleja que un avión de combate de hace sólo 14 años, gestionado por un software que requiere más de 10 millones de líneas de código, frente a los 1,7 millones de líneas de código que requería un avión de combate en 1997.

La sociedad industrial que surgió en el siglo XIX y que perduró durante la mayor parte del siglo XX ha dado lugar a la actual "sociedad de la información", con una creciente capacidad tecnológica para almacenar cada vez más información y hacerla circular cada vez más rápidamente.

Si hacemos caso de la predicción realizada por Peter Drucker en los años 90, esta sociedad de la información debería dar paso a una auténtica sociedad del conocimiento.

Una sociedad en la que toda esta gran cantidad de información en circulación pueda transformarse en verdadero conocimiento, capaz de producir a su vez más conocimiento, para lograr un desarrollo económico sostenible.

Pero no hay que olvidar, que todo el aprendizaje y el conocimiento que podamos generar, no se podrá transformar realmente en innovación y competitividad, si no contamos también con personas emprendedoras, como las que han ayudado a situar a este país en la novena posición de la economía mundial, una posición que deberíamos tratar de consolidar, e incluso de mejorar. 
En definitiva, nos enfrentamos a un mundo muy competitivo, pero también lleno de oportunidades, para los individuos y los países que sepan aprovecharlas con ima- ginación y nuevas ideas. Desde la universidad, y también desde las empresas, tenemos que ser capaces de impulsarlas.

\section{BIBLIOGRAFÍA}

Círculo de Empresarios, CEOE (2011): Documento conjunto Círculo de Empresarios-CEOE sobre la competitividad de la industria española.

Consejo Empresarial para la Competitividad (2011): Fortalezas y perspectivas de la economía española.
Fundación Cotec para la Innovación Tecnológica (2011): Tecnología e innovación en España. Informe COTEC 2011.

Fundación CYD (2011): Informe CYD 2010.

Ministerio de Educación (2010): Panorama de la educación. Indicadores de la OCDE 2010. Informe español.

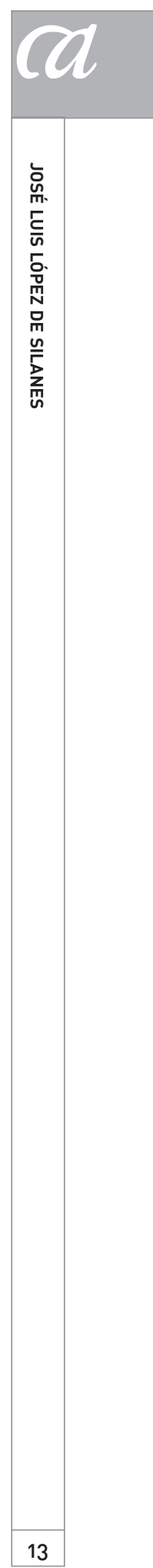

